\title{
Antimicrobial stewardship in remote primary healthcare across northern Australia
}

\author{
Will Cuningham ${ }^{1}$, Lorraine Anderson ${ }^{2}$, Asha C Bowen ${ }^{1,3,4}$, Kirsty Buising ${ }^{5,6}$, Christine Connors ${ }^{7}$, Kathryn Daveson \\ $8,9,10$, Joanna Martin ${ }^{2}$, Stacey McNamara ${ }^{9}$, Bhavini Patel ${ }^{7,11}$, Rod James $^{5}$, John Shanks ${ }^{7}$, Kerr Wright ${ }^{2}$, Trent \\ Yarwood $^{9,12,13,14}$, Steven YC Tong ${ }^{1,15}$, Jodie McVernon ${ }^{\text {Corresp. } 5,16}$ \\ ${ }^{1}$ Menzies School of Health Research, Charles Darwin University, Darwin, Northern Territory, Australia \\ 2 Kimberley Aboriginal Medical Services, Kimberley, Western Australia, Australia \\ 3 Wesfarmers Centre for Vaccines and Infectious Diseases, Telethon Kids Institute, University of Western Australia, Perth, Western Australia, Australia \\ 4 Department of Infectious Diseases, Perth Children's Hospital, Perth, Western Australia, Australia \\ 5 The Peter Doherty Institute for Infection and Immunity, The Royal Melbourne Hospital and The University of Melbourne, Melbourne, Victoria, Australia \\ 6 Victorian Infectious Diseases Service, The Royal Melbourne Hospital, Melbourne, Victoria, Australia \\ 7 Top End Health Service, NT Department of Health, Darwin, Northern Territory, Australia \\ 8 Antimicrobial Use and Resistance in Australia Project, Australian Commission for Safety and Quality in Healthcare, Canberra, Australian Capital Territory, \\ Australia \\ 9 Queensland Statewide Antimicrobial Stewardship Program, Metro North Hospital and Health Service, Brisbane, Queensland, Australia \\ 10 Department of Infectious Diseases and Microbiology, Canberra Hospital, Canberra, Australian Capital Territory, Australia \\ 11 Charles Darwin University, Darwin, Northern Territory, Australia \\ 12 Cairns Hospital, Cairns, Queensland, Australia \\ 13 Rural Clinical School, University of Queensland, Brisbane, Queensland, Australia \\ 14 College of Medicine and Dentistry, James Cook University, Townsville, Queensland, Australia \\ 15 Victorian Infectious Diseases Service, The Royal Melbourne Hospital, and Doherty Department University of Melbourne, at the Peter Doherty Institute \\ for Infection and Immunity, Melbourne, Victoria, Australia \\ 16 Melbourne School of Population and Global Health, The University of Melbourne, Melbourne, Victoria, Australia \\ Corresponding Author: Jodie McVernon \\ Email address: j.mcvernon@unimelb.edu.au
}

\section{Background}

The high burden of infectious disease and associated antimicrobial use likely contribute to the emergence of antimicrobial resistance (AMR) in remote Australian Aboriginal communities. We aimed to develop and apply context-specific tools to audit antimicrobial use in the remote primary healthcare setting.

\section{Methods}

We adapted the General Practice version of the National Antimicrobial Prescribing Survey (GP NAPS) tool to audit antimicrobial use over 2-3 weeks in fifteen remote primary healthcare clinics across the Kimberley region of Western Australia (03/2018-06/2018), Top End of the Northern Territory (08/2017-09/2017) and far north Queensland (05/2018-06/2018). At each clinic we reviewed consecutive clinic presentations until 30 presentations where antimicrobials had been used were included in the audit. Data recorded included the antimicrobials used, indications and treating health professional. We assessed the appropriateness of antimicrobial use and functionality of the tool. 


\section{Results}

We audited the use of 668 antimicrobials. Skin and soft tissue infections were the dominant treatment indications (WA: 35\%; NT: 29\%; QLD: 40\%). Compared with other settings in Australia, narrow spectrum antimicrobials like benzathine benzylpenicillin were commonly given and the appropriateness of use was high (WA: 91\%; NT: 82\%; QLD: 65\%). While the audit was informative, non-integration with practice software made the process manually intensive.

\section{Conclusions}

Patterns of antimicrobial use in remote primary care are different from other settings in Australia. The adapted GP NAPS tool functioned well in this pilot study and has the potential for integration into clinical care. Regular stewardship audits would be facilitated by improved data extraction systems. 


\section{Antimicrobial stewardship in remote primary}

\section{2 healthcare across northern Australia}

3

Will Cuningham ${ }^{1}$, Lorraine Anderson ${ }^{2}$, Asha Bowen ${ }^{1,3,4}$, Kirsty Buising ${ }^{5,6}$, Christine Connors ${ }^{7}$, Kathryn Daveson ${ }^{8-10}$, Joanna Martin ${ }^{2}$, Stacey McNamara ${ }^{9}$, Bhavini Patel ${ }^{7,11}$, Rod James ${ }^{5}$, John Shanks $^{7}$, Kerr Wright ${ }^{2}$, Trent Yarwood ${ }^{9,12-14}$, Steven Tong ${ }^{15,1}$, Jodie McVernon ${ }^{5,16}$

${ }^{1}$ Menzies School of Health Research, Charles Darwin University, Darwin, Northern Territory, Australia

${ }^{2}$ Kimberley Aboriginal Medical Services, Kimberley, Western Australia, Australia

${ }^{3}$ Wesfarmers Centre for Vaccines and Infectious Diseases, Telethon Kids Institute, University of Western Australia, Perth, Western Australia, Australia

${ }^{4}$ Department of Infectious Diseases, Perth Children's Hospital, Perth, Western Australia, Australia

${ }^{5}$ The Peter Doherty Institute for Infection and Immunity, The Royal Melbourne Hospital and The University of Melbourne, Melbourne, Victoria, Australia

${ }^{6}$ Victorian Infectious Diseases Service, The Royal Melbourne Hospital, Melbourne, Victoria, Australia

${ }^{7}$ Top End Health Service, NT Department of Health, Darwin, Northern Territory, Australia ${ }^{8}$ Antimicrobial Use and Resistance in Australia Project, Australian Commission for Safety and Quality in Healthcare, Canberra, Australian Capital Territory, Australia

${ }^{9}$ Queensland Statewide Antimicrobial Stewardship Program, Metro North Hospital and Health Service, Brisbane, Queensland, Australia

${ }^{10}$ Department of Infectious Diseases and Microbiology, Canberra Hospital, Canberra, Australian Capital Territory, Australia

${ }^{11}$ Charles Darwin University, Darwin, Northern Territory, Australia

${ }^{12}$ Cairns Hospital, Cairns, Queensland, Australia

${ }^{13}$ Rural Clinical School, University of Queensland, Brisbane, Queensland, Australia

${ }^{14}$ College of Medicine and Dentistry, James Cook University, Townsville, Queensland, Australia

${ }^{15}$ Victorian Infectious Diseases Service, The Royal Melbourne Hospital, and Doherty Department University of Melbourne, at the Peter Doherty Institute for Infection and Immunity, Melbourne, Victoria, Australia

${ }^{16}$ Melbourne School of Population and Global Health, The University of Melbourne, Melbourne, Victoria, Australia

Corresponding Author:

Jodie McVernon ${ }^{5,16}$

792 Elizabeth St, Melbourne, Victoria, 3000, Australia 
41

42

Email address: j.mcvernon@,unimelb.edu.au

\section{Abstract}

\section{Background}

The high burden of infectious disease and associated antimicrobial use likely contribute to the emergence of antimicrobial resistance (AMR) in remote Australian Aboriginal communities. We aimed to develop and apply context-specific tools to audit antimicrobial use in the remote primary healthcare setting.

\section{Methods}

We adapted the General Practice version of the National Antimicrobial Prescribing Survey (GP NAPS) tool to audit antimicrobial use over 2-3 weeks in fifteen remote primary healthcare clinics across the Kimberley region of Western Australia (03/2018-06/2018), Top End of the Northern Territory (08/2017-09/2017) and far north Queensland (05/2018-06/2018). At each clinic we reviewed consecutive clinic presentations until 30 presentations where antimicrobials had been used were included in the audit. Data recorded included the antimicrobials used, indications and treating health professional. We assessed the appropriateness of antimicrobial use and functionality of the tool.

\section{Results}

We audited the use of 668 antimicrobials. Skin and soft tissue infections were the dominant treatment indications (WA: 35\%; NT: 29\%; QLD: 40\%). Compared with other settings in Australia, narrow spectrum antimicrobials like benzathine benzylpenicillin were commonly given and the appropriateness of use was high (WA: 91\%; NT: 82\%; QLD: 65\%). While the audit was informative, non-integration with practice software made the process manually intensive.

\section{Conclusions}

Patterns of antimicrobial use in remote primary care are different from other settings in Australia. The adapted GP NAPS tool functioned well in this pilot study and has the potential for integration into clinical care. Regular stewardship audits would be facilitated by improved data extraction systems.

\section{Introduction}

Antimicrobial resistance (AMR) is a major healthcare issue. Compared with other countries, Australia currently has relatively low (albeit rising) rates of AMR, despite comparatively high rates of antimicrobial use in humans. ${ }^{1-4}$ Remote regions in northern Australia, however, have some of the highest rates of AMR in the world; for example, at least $40 \%$ of Staphylococcus aureus community-associated isolates are methicillin-resistant (MRSA). ${ }^{5-7}$ In comparison, rates of MRSA are about 15-20\% elsewhere in Australia. Recognising the threat of emerging resistance to human health, the Australian Government Departments of Health and Agriculture published the first National Antimicrobial Resistance Strategy in June 2015. ${ }^{8}$ The accompanying implementation plan highlighted areas for specific focus including identification of barriers and 
80

81

82

83

84

85

86

87

88

89

90

91

92

93

94

95

96

97

98

99

100

101

102

103

104

105

106

107

108

109

110

111

112

113

114

115

116

117

118

119

enablers to antimicrobial stewardship (AMS) in Indigenous healthcare organisations to inform development of setting-specific resources. ${ }^{9}$ Addressing AMR in Indigenous primary healthcare and coordinating AMS programs in partnership with Aboriginal and Torres Strait Islander communities have been highlighted as priorities. ${ }^{10}$

Australia is a geographically large country. Northern Australia alone (the region north of the Tropic of Capricorn) is approximately equivalent in area to Argentina and is sparsely populated. The Northern Territory (NT), for example, has a population density of 0.2 people $/ \mathrm{km}^{2}$, the lowest of Australia's eight jurisdictions. ${ }^{11}$ Of its 245,000 residents, 30\% live in rural areas (compared with $10 \%$ Australia-wide). ${ }^{12}$ Furthermore, while only $3 \%$ of the Australian population are Aboriginal and/or Torres Strait Islander, this is 30\% in the NT. ${ }^{13}$ Of these Aboriginal and Torres Strait Islander peoples, $81 \%$ live remotely (calculated using an index reflecting the community's population size and level of access to goods and services), often many hundreds, if not thousands of kilometres from the nearest urban center (Figure 1). In northern WA and Queensland, there are similar sparsely populated communities. These communities have small healthcare clinics, often Aboriginal Community Controlled Health Services, and antimicrobials are routinely supplied through the Pharmaceutical Benefits Scheme (PBS). The health workforce is varied, comprised of doctors, nurses, Aboriginal Health Practitioners and other health workers, many of whom are on temporary contracts or work on a fly-in/fly-out basis. The mean annual staff turnover rate in this setting is as high as $128 \%{ }^{14}$ Usually more severe cases of infection require treatment at a larger, urban hospital which involves air transport.

The high burden of infectious diseases in remote communities necessitates high antimicrobial use, emphasising the need for good AMS. ${ }^{15}$ However, specific information about prescribing in remote settings is difficult to obtain. For example, the emergence of trimethoprim-resistant clones of community associated MRSA in the Northern Territory ${ }^{16}$, Western Australia (WA) ${ }^{17}$ and Queensland (QLD) ${ }^{18}$ may be related to recommendations for co-trimoxazole in skin sore treatment ${ }^{19}$, but this hypothesis cannot be tested due to a lack of prescribing data.

Hospital-based AMS programs are tasked with monitoring antimicrobial use and implementing AMS procedures in northern Australian hospitals (Kimberley Aboriginal Health Planning Forum (KAHPF) stewardship committee, WA; Top End Health Service (TEHS) stewardship committee, NT; Queensland Statewide Antimicrobial Stewardship Program (QSAMSP)) to meet accreditation standards. ${ }^{20,21}$ Extension of stewardship into the remote primary healthcare sector has been minimal due to a lack of related accreditation requirements and audit tools, limited workforce and competing priorities. Authorised healthcare workers other than doctors, such as registered nurses and Aboriginal Health Practitioners may supply and administer antimicrobials in remote settings, in accordance with local treatment protocols and jurisdictional legislation. ${ }^{22-24}$ Context-specific tools are needed to capture prescribing practices and provide evidence-based feedback and indicators to support best clinical practice and health outcomes.

Hospital-based National Antimicrobial Prescribing Surveys (NAPS) have been conducted since 2011 and provide a yearly snapshot of antimicrobial use, indications and appropriateness in reference to local and national guidelines. ${ }^{25}$ Participation in the NAPS is voluntary, 314 hospitals 
120

121

122

123

124

125

126

127

128

129

130

131

132

133

134

135

136

137

138

139

140

141

142

143

144

145

nation-wide participating in the latest report. Appropriateness of antimicrobial use in the hospital setting was $76 \%$, cefazolin, ceftriaxone and amoxicillin-clavulanate being the most commonly used. ${ }^{25,26}$ Other studies have highlighted some differences in antimicrobial use and challenges to AMS in the regional and remote hospital setting. ${ }^{27-30}$ Related AMS tools are being developed to assess antimicrobial prescribing practices in urban General Practices (GP). Data on antimicrobial use in GP is limited, particularly in remote primary healthcare. Some data collected through the PBS and GP electronic software show the frequent use of penicillins with extended spectrum and $\beta$-lactamase inhibitor combinations. ${ }^{5}$ Rates of antimicrobial use have decreased in recent years, but there continues to be overuse compared with guideline recommendations and inappropriate use of antibiotics for respiratory indications like acute bronchitis and influenza. ${ }^{5}$ Crucially, antimicrobial use in the remote northern Australian setting is not captured by the PBS, leaving a gap in our understanding of antimicrobial use. Given the primary healthcare setting of this audit, we adapted and piloted the GP version of the NAPS tool (GP NAPS) in remote clinics in northern WA, NT and Queensland.

Our aims were to:

1. Develop and pilot an audit tool of antimicrobial use for use in a remote primary healthcare setting

2. In piloting the tool across 15 primary healthcare clinics in 3 jurisdictions, we sought to describe:

a. Similarities and differences in prescribing patterns by jurisdiction and healthcare professional status.

b. Appropriateness of prescribing.

c. Functionality of the audit tool in a qualitative sense

The following Materials \& Methods section describes the process taken to achieve aim 1, and the Results section presents the descriptive analysis for aim 2.

\section{Materials \& Methods}

\section{Adaption of the GP NAPS tool}

Through an iterative process the study team, a multidisciplinary group of researchers, modified the exiting GP NAPS audit tool. Adapted data fields included additions that reflect the health professional types, antimicrobials and indications common in remote primary healthcare clinics, while removing fields that were not relevant. It was designed to provide consistent data interpretation across the different states and territories and by various auditor types (Appendix A).

The study team comprised:

1. Clinical pharmacists working within the jurisdictions

2. National Centre for Antimicrobial Stewardship (NCAS) staff with experience in developing and using the GP NAPS audit tool

3. Infectious diseases clinicians with expertise in antimicrobial stewardship or working in remote health 
4. Individuals with key oversight roles for remote health The study team provided relevant information on the differences between the various proposed primary healthcare clinics and possible requirements for additions or alterations to the GP NAPS audit tool. This required several rounds of template design and was conducted via email and teleconferences between 16/10/2017 to 26/03/2018.

Criteria used to assess appropriateness of antimicrobial use were identical to those used in the GP NAPS tool but tailored to local guidelines (Appendix B). In addition to the national Therapeutic Guidelines ${ }^{31}$ (to which access and promotion of are requirements of the National Safety and Quality Health Service Standards ${ }^{21}$ (NSQHS)), there are three regionally specific clinical guidelines used across northern Australia: the Kimberley Clinical Protocols and Guidelines (KCPG) in northern WA ${ }^{22}$, the Central Australian Rural Practitioners Association Standard Treatment Manual (CARPA) in the $\mathrm{NT}^{23}$ and the Primary Clinical Care Manual (PCCM) in Queensland. ${ }^{24}$ To assess variation in these guidelines, recommended antibiotic treatments for selected indications were compared (Table S1).

In this report we refer to antimicrobial 'use' instead of 'prescription' to reflect the range of health professionals who are legally able to supply and administer antimicrobials in the remote setting, specifically including doctors, remote area nurses and Aboriginal Health Practitioners. Selection of study sites

Five primary healthcare clinics of varying capacities were selected by local project leads in each of the three northern jurisdictions. These clinics were governed by the Kimberley Aboriginal Medical Services (KAMS) in WA, TEHS in the NT and Torres and Cape Hospital and Health Service (TCHHS) in Queensland.

\section{Audit process}

Following remote training by NCAS, local auditors reviewed consecutive clinic presentations to identify the first 30 resulting in use of at least one antibiotic, antiviral, antifungal or antiparasitic agent. The same patient could be included more than once if treatment had not previously been given for the same indication.

Data were collected over a 2- to 3-week period via a standardised paper form (Appendix A) and later transferred to a Microsoft Excel (Office 365) spreadsheet. Auditors in the Kimberley and NT used remote access to electronic health records while in Queensland medical records were reviewed on site. All auditors were employees of the involved health services, familiar with local treatment guidelines.

Guideline compliance and appropriateness of each antimicrobial used was assessed against agreed definitions (Appendix B) ${ }^{25}$, based on the NSQHS and Antimicrobial Stewardship Clinical Care Standard. ${ }^{32}$ In brief, antimicrobial use was "Appropriate" if it followed the local or Therapeutic Guidelines, or was endorsed by an infectious disease expert or clinical microbiologist, or was the most narrow-spectrum antimicrobial that covers the causative pathogen, or was a reasonable alternative to the guidelines (usually with justification for the choice documented in the notes). The alternative category, "Inappropriate", included antimicrobials used at the incorrect dose or too broad spectrum for the indication, or the choice 
200

201

202

203

204

205

206

207

208

209

210

211

212

213

214

215

216

217

218

219

220

221

222

223

224

225

226

227

228

229

230

231

232

233

234

235

236

237

238

239

was unlikely to treat the causative pathogen, or the indication didn't require antimicrobial treatment at all. The category "Not Assessable" was used if there was insufficient recorded information. The NAPS team at NCAS provided phone and email support to the local audit teams. Ten percent of antimicrobials audited in each facility were randomly reviewed by an expert at NCAS to assess reliability and validity of local scoring.

Data analysis

The three jurisdictional datasets were transferred to a secure central repository and merged into a single dataset for analysis in Stata 15.1. ${ }^{33}$ Descriptive analyses of the data were conducted and presented as proportions for categorical variables and medians for continuous data. No inferential statistical analyses were performed for this pilot study.

Qualitative survey of end user functionality of the GP NAPS tool

A written questionnaire was used to survey local auditors to assess the practicality, usability, feasibility, and generalisability of the audit tool to evaluate its suitability in context (Table S2).

We did not aim to test the construct validity or reliability of the tool.

Ethical approval

Ethical approval was obtained from each jurisdictional ethics committee (Western Australia Aboriginal Health Ethics Committee (WAAHEC): 839; Top End Health Research Ethics Committee (TEHREC): 2017-3012; Far North Queensland Human Research Ethics Committee (FNQ HREC): HREC/18/QCH/3, PHA RD007329, SSA/18/FNQ/9,10,11,12,13,14). A waiver of consent was granted because this research was retrospective, used de-identified data routinely collected at the time of healthcare provision and thus carried no risk to participants.

\section{Results}

\section{Cohort characteristics}

668 antimicrobials for 529 patients between August 2017 and June 2018 were included (Table 1). The median age of patients was 23 years (interquartile range: $7-45$ years), with $20 \%$ below 5 years. Females comprised $60 \%$ of patients. As expected, most patients were Aboriginal \& Torres Strait Islander people.

\section{Trends in antimicrobial use}

Nurses $(305 / 668,46 \%)$ and doctors $(347 / 668,52 \%)$ supplied/administered most of the antimicrobials with some variation across jurisdictions (Table 2). Aboriginal \& Torres Strait Islander Health Practitioners rarely supplied/administered antimicrobials $(4 / 668,<1 \%)$. This is generally reflective of the staff composition, although we might expect a higher proportion of Aboriginal Health Practitioners given that they comprise up to $10 \%$ of the workforce. ${ }^{34}$, ${ }^{35}$ Skin and soft tissue infections (SSTI) were the most common indication for antimicrobial use in all jurisdictions (WA: 35\%; NT: 29\%; QLD: 40\%). The most frequently used antibiotics were amoxicillin and benzathine benzylpenicillin. Co-trimoxazole was more commonly used in Queensland than other states (WA: 7\%; NT: 5\%; QLD: 18\%).

Figure 2 reflects how different presentations are managed. In WA and the NT, SSTIs made up a slightly larger proportion of cases treated by nurses ( $42 \%$ and $32 \%$ respectively) compared with 
240

241

242

243

244

245

246

247

248

249

250

251

252

253

254

255

256

257

258

259

260

261

262

263

264

265

266

267

268

269

270

271

272

273

274

275

276

277

278

279

doctors (29\% and 24\% respectively). Furthermore, lower respiratory tract infections (LRTI) were treated by only doctors in WA, but by doctors and nurses in the NT. Secondary prophylaxis for rheumatic heart disease was used by only doctors in Queensland (14/14), by mostly nurses in the NT (18/22) and by doctors (3/6), nurses (2/6) and Aboriginal and Torres Strait Islander Health Practitioners (1/6) in WA (not shown in Figure 2).

For SSTIs (mainly impetigo and folliculitis/abscess) in WA and the NT, benzathine benzylpenicillin was more commonly used by nurses ( $43 \%$ and $29 \%$ respectively) than by doctors (24\% and 17\% respectively) (Figure 3). Co-trimoxazole was used more in Queensland $(43 \%)$ than in WA $(16 \%)$ and the NT $(14 \%)$, especially by doctors.

The second most common syndrome leading to antimicrobial use in the NT was LRTI (18\%; WA: 8\%, QLD: 4\%). Remote Area Nurses treated most LRTIs in the NT (63\%), using procaine penicillin (most often for pneumonia) or azithromycin (bronchiectasis) for $41 \%$ (Figure S1). Sexually transmitted infections were common in both WA and Queensland $(10 \%$ and $12 \%$ respectively; NT: 3\%), with azithromycin frequently used (WA: 42\%; QLD: 43\%; NT: none), however there was notably more ceftriaxone used in Queensland (WA: 4\%; QLD: 25\%; NT: none) (Figure S2).

\section{Appropriateness of antimicrobial use}

More than $85 \%$ of antimicrobials used were considered appropriate in WA and the NT according to local or Therapeutic Guidelines (205/225 and 159/193 respectively) (Figure 4). In Queensland, $65 \%(147 / 226)$ of antimicrobials used were considered appropriate. On average, $86 \%$ of antimicrobials used by nurses were appropriate (WA: 91\% (90/99); NT: 87\% (122/141); QLD: $77 \%(47 / 61)$ ) (Table S3), compared with $73 \%$ of those used by doctors (WA: $90 \%$; NT: 73\%; QLD: 61\%).

In all jurisdictions, dose and frequency of antimicrobial treatment were the most commonly incorrect features of antimicrobial treatment (WA: 8\%; NT: 7\%; QLD: 1\%) (Table S4). Other commonly incorrect features included antimicrobials used for an indication that did not require antimicrobial treatment (WA: 4\%; NT: 7\%; QLD: 10\%), or with a duration that extended beyond the recommended period (WA: 7\%; NT: 2\%; QLD: 4\%).

Antimicrobials used for trauma and non-surgical wound infections contributed substantially to inappropriate use by doctors in Queensland with 7/24 deemed appropriate (WA: 10/14; NT: 1/5). For wound infections, co-trimoxazole was used in 10 of 15 cases in Queensland (0/10 appropriate due to incorrect spectrum of activity and/or incorrect dosing when compared to the local or national guidelines) and in 4 of 7 in WA (4/4 appropriate). Overall, antimicrobials used for impetigo were mostly appropriate (WA: 17/18; NT: 9/10; QLD: 15/21) for which cotrimoxazole was used more often in Queensland (WA: 1/1 appropriate; NT: 2/2 appropriate; QLD: 9/10 appropriate) compared with benzathine benzylpenicillin in WA (16/16 appropriate) and the NT (7/8 appropriate).

Amoxicillin was often used inappropriately in Queensland by doctors for otitis media due to incorrect dose or frequency (WA: 3/3 (two nurses) appropriate; NT: 8/9 (all nurses) appropriate; QLD: 1/7 appropriate). Amoxicillin and amoxicillin-clavulanate were also used inappropriately 
280 for pneumonia in Queensland (WA: 5/5 appropriate; NT: 7/12 appropriate; QLD: 0/5

281 appropriate).

282 Qualitative survey of end user functionality of the GP NAPS tool

283 While the tool was found to be acceptable in this context, auditors made some recommendations

284 for improvement (Table S2), such as the addition of some indications common in the remote

285 primary healthcare setting and training specific to the assessment of antimicrobials used in these

286 cases to improve consistency across jurisdictions. An additional category to grade antimicrobials

287 compliant with national guidelines but not with local guidelines was suggested as being useful

288 for future analyses and to identify variations in guidelines. Likewise, stratifying by medical

289 onsite and offsite (i.e. telephone order) review was suggested. Auditors reported a lack of detail

290 in many clinical records, including absence of imaging, laboratory results and other patient

291 medical history, which made identification of the indication and assessment of appropriateness

292 difficult in some cases, especially at clinics that used only paper-based medical records.

293 However, this is a challenge faced in any type of survey using administrative data, rather than a

294 shortcoming of the tool itself, and is a common limitation of AMS in general. ${ }^{36}$ The audit

295 findings were reported back to each jurisdiction promptly which enabled identification of action

296 areas for their AMS programs. Auditors also highlighted areas in regional guidelines that could

297 be refined to improve interpretation by less locally experienced staff and where guidelines could

298 be updated to better align with the national Therapeutic Guidelines where appropriate.

299

300

301

\section{Discussion}

Our primary aim was to develop and pilot an audit tool of antimicrobial use for the remote primary healthcare setting in Australia. Adaptations made to an existing tool for urban primary healthcare use were relatively minor, incorporating health professionals, antimicrobials and indications common in remote primary healthcare as well as the Indigenous status of the patient. We gained useful preliminary insights on antimicrobial use, however these are descriptive findings that will require confirmation in future studies given that this audit included only a select few communities across northern Australia. Support from an expert team (at NCAS) was required to ensure consistency in data quality and interpretation. Overall the tool was suitable for use and was well received by the local health services in their first access to an audit tool. The data demonstrated that SSTIs were the most frequent indications for antimicrobial use in all three regions. ${ }^{15,37}$ This finding highlights the difference in morbidity profiles and patterns of antimicrobial use between remote and urban communities, as upper respiratory tract infections are typically the most common reason for an antimicrobial prescription in urban areas. ${ }^{38}$ The appropriateness of antimicrobial use was high compared with urban GP settings around Australia. We found that narrow spectrum antibiotics like benzathine benzylpenicillin were frequently used in the remote setting, in contrast to the broader spectrum antibiotics like cephalexin and amoxicillin-clavulanate that are more often prescribed in urban primary healthcare. ${ }^{39}$ Strong local ownership of region-specific guidelines combined with regulatory mechanisms to regulate the supply and provision of antimicrobials may have supported more 
320

321

322

323

324

325

326

327

328

329

330

331

332

333

334

335

336

337

338

339

340

341

342

343

344

345

346

347

348

349

350

351

352

353

354

355

356

357

358

standardized antimicrobial use. Furthermore, a higher rate of monthly benzathine benzylpenicillin injections for the treatment and prevention of rheumatic heart disease may increase overall appropriateness compared with other parts of Australia that have a proportionally smaller Indigenous population.

An important difference is that the health professionals supplying and administering antimicrobials differ in remote areas from urban settings. This study showed that nurses undertook a substantial proportion of this activity in remote areas whereas this is uncommon in primary care in urban areas. Antimicrobial use by Aboriginal \& Torres Strait Islander Health Practitioners/Workers were lower than anticipated and further work is required to understand the barriers to antimicrobial use by this group. ${ }^{40-42}$

Conflicts between multiple guidelines can influence antimicrobial use, and this issue can be exacerbated by high staff turnover rates in the remote setting. ${ }^{14}$ For example, at the time of the audit, the regional Queensland guidelines (PCCM) included co-trimoxazole for impetigo but not for folliculitis/abscess, while the KCPG and CARPA include co-trimoxazole for both conditions. Similarly, co-trimoxazole for cellulitis was assessed as adequate treatment (i.e. in line with currently accepted medical practice) but non-compliant with the Therapeutic Guidelines, PCCM or other local guidelines. Some of these inconsistences have been resolved with release of the $10^{\text {th }}$ edition of the PCCM in 201924, which lists co-trimoxazole as indicated for impetigo and folliculitis/abscess, and for cellulitis in the Therapeutic Guidelines for people with an increased risk of MRSA infection. ${ }^{31}$ In future, electronic guidelines or decision support tools could be updated in a more timely manner.

Next steps

This project aligns with the Australian Commission on Safety and Quality in Health Care's objectives of setting safety and quality goals for Aboriginal and Torres Strait Islander people in health service organisations, specifically agreeing on measures to analyse health outcomes and measuring performance towards targets. ${ }^{43,44}$ Embedding this research within existing services facilitates translation of research findings into ongoing practice and capacity building in AMS across remote clinics.

Changes to electronic software in the practice to improve indication documentation would make future auditing activity more efficient. ${ }^{36}$ Automated data extraction and analysis would support remote AMS audits, improve assessment of antimicrobial use and understanding of the epidemiology of community-acquired infections, and could be linked to decision support tools. Real-time antimicrobial use data would complement other recent efforts in AMR surveillance across northern Australia (HOTspots: amrhotspots.com.au) and support clinical and public health efforts that aim to improve clinical care and reduce the emergence of AMR. A corresponding investment in workforce capacity in AMS is needed, across all relevant practitioner groups. Local Aboriginal engagement and leadership is essential in addressing AMR, the underlying burden of disease perpetuated by social determinants, and to prioritise the implementation of ongoing AMS in this setting. Furthermore, a focus on AMS in the accreditation standards of 
359 primary healthcare facilities is needed to enhance AMS activities and drive progress at the 360 systems level.

361

362 Conclusions

363 The development and piloting of an audit tool for antimicrobial use revealed that the morbidity

364 profile and antimicrobial use in remote Aboriginal primary healthcare clearly differ compared

365 with other primary care settings in Australia. The manual process of data collection was resource

366 intensive. Broader implementation and scale up will likely require improved methods for

367 automated data extraction.

368

369

Acknowledgements

370 The authors acknowledge and thank the primary healthcare clinics and staff who participated in

371 this project, as well as NCAS for their support. We also thank Ms Amy Legg for her role as

372 Project Officer. 


\section{References}

375 1. Cecchini M, Langer J, Slawomirski L. Antimicrobial Resistance in G7 Countries and Beyond:

376 Economic Issues, Policies and Options for Action. Organisation for Economic Co-operation and 377 Development. 2015.

378 2. Antimicrobial resistance, Policy Insights. Organisation for Economic Co-operation and 379 Development. 2016.

380 3. Klein EY, Van Boeckel TP, Martinez EM, Pant S, Gandra S, Levin SA, Goossens H, Laxminarayan R. 381 Global increase and geographic convergence in antibiotic consumption between 2000 and 2015.

382 Proceedings of the National Academy of Sciences of the United States of America. 2018;115(15):E3463383 E70.

384 4. Van Boeckel TP, Gandra S, Ashok A, Caudron Q, Grenfell BT, Levin SA, Laxminarayan R. Global 385 antibiotic consumption 2000 to 2010: an analysis of national pharmaceutical sales data. The Lancet 386 Infectious Diseases. 2014;14(8):742-50.

387 5. Australian Commission on Safety and Quality in Health Care (ACSQHC). AURA 2019: third 388 Australian report on antimicrobial use and resistance in human health. Sydney: ACSQHC; 2019.

3896 6ustralian Commission on Safety and Quality in Health Care. Australian Passive Antimicrobial 390 Resistance Surveillance. First report: multi-resistant organisms. Sydney: ACSQHC; 2018.

3917 7. Tong SY, McDonald MI, Holt DC, Currie BJ. Global implications of the emergence of community392 associated methicillin-resistant Staphylococcus aureus in Indigenous populations. Clinical infectious 393 diseases : an official publication of the Infectious Diseases Society of America. 2008;46(12):1871-8.

3948 8 Responding to the threat of antimicrobial resistance: Australia's First National Antimicrobial

395 Resistance Strategy 2015-2019. Australian Government Departments of Health and Agriculture, 396 Commonwealth of Australia. 2015.

397 9. Implementation Plan: Australia's First National Antimicrobial Resistance Strategy 2015-2019.

398 Australian Government Department of Health and Department of Agriculture and Water Resources, 399 Commonwealth of Australia. 2016.

400 10. Bowen AC, Daveson K, Anderson L, Tong SY. An urgent need for antimicrobial stewardship in 401 Indigenous rural and remote primary health care. The Medical journal of Australia. 2019;211(1):9-11. e1.

402 11. Australian Bureau of Statistics, 2016, 3218.0 - Regional Population Growth, Australia, viewed 403 28/04/2020,

404 https://www.abs.gov.au/ausstats/abs@.nsf/Previousproducts/3218.0Main\%20Features752016.

405 12. Australian Bureau of Statistics, 2019, 3101.0 - Australian Demographic Statistics, viewed 406 28/04/2020,

407 https://www.abs.gov.au/AUSSTATS/abs@.nsf/DetailsPage/3101.0Sep\%202019?OpenDocument.

408 13. Australian Bureau of Statistics, 2016, 3238.0.55.001 - Estimates of Aboriginal and Torres Strait 409 Islander Australians, viewed 28/04/2020, 410 https://www.abs.gov.au/ausstats/abs@.nsf/mf/3238.0.55.001.

411 14. Russell DJ, Zhao Y, Guthridge S, Ramjan M, Jones MP, Humphreys JS, Wakerman J. Patterns of 412 resident health workforce turnover and retention in remote communities of the Northern Territory of 413 Australia, 2013-2015. Hum Resour Health. 2017;15(1):52.

414 15. Cuningham W, McVernon J, Lydeamore MJ, Andrews RM, Carapetis J, Kearns T, Clucas D, 415 Gundjirryirr Dhurrkay R, Tong SYC, Campbell PT. High burden of infectious disease and antibiotic use in 416 early life in Australian Aboriginal communities. Aust N Z J Public Health. 2019;43(2):149-55.

417 16. Tong SY, Varrone L, Chatfield MD, Beaman M, Giffard PM. Progressive increase in community418 associated methicillin-resistant Staphylococcus aureus in Indigenous populations in northern Australia 419 from 1993 to 2012. Epidemiology and infection. 2015;143(7):1519-23. 
420 17. Coombs G, Pearson J, Robinson O. Western Australian methicillin-resistant Staphylococcus

421

422

423

424

425

426

427

428

429

430

431

432

433

434

435

436

437

438

439

440

441

442

443

444

445

446

447

448

449

450

451

452

453

454

455

456

457

458

459

460

461

462

463

464

465

466 aureus (MRSA) epidemiology and typing report. Perth, Australia. 2015.

18. Guthridge I, Smith S, Horne P, Hanson J. Increasing prevalence of methicillin-resistant Staphylococcus aureus in remote Australian communities: implications for patients and clinicians. Pathology. 2019;51(4):428-31.

19. Bowen AC, Tong SYC, Andrews RM, O'Meara IM, McDonald MI, Chatfield MD, Currie BJ, Carapetis JR. Short-course oral co-trimoxazole versus intramuscular benzathine benzylpenicillin for impetigo in a highly endemic region: an open-label, randomised, controlled, non-inferiority trial. The Lancet. 2014;384(9960):2132-40.

20. Australian Commission on Safety and Quality in Health Care. National Safety and Quality Health Service Standards guide for hospitals. Sydney: ACSQHC. 2017.

21. Australian Commission on Safety and Quality in Health Care. National Safety and Quality Health Service Standards. 2nd ed. Sydney: ACSQHC. 2017.

22. Kimberley Clinical Protocols and Guidelines (KCPG). 2016; https://www.kahpf.org.au/clinicalprotocols (date accessed: 01/10/2019).

23. Remote Primary Health Care Manuals. (2017). CARPA Standard Treatment Manual (7th edition). Alice Springs, NT: Centre for Remote Health.

24. Queensland Health, Royal Flying Doctor Service (Queensland Section), Primary Clinical Care Manual 10th edition 2019, the Rural and Remote Clinical Support Unit, Torres and Cape Hospital and Health Service, Cairns.

25. National Centre for Antimicrobial Stewardship and Australian Commission on Safety and Quality in Health Care. Antimicrobial prescribing practice in Australian hospitals: results of the 2017 Hospital National Antimicrobial Prescribing Survey. Sydney: ACSQHC; 2018.

26. SA Health, Australian Commission on Safety and Quality in Health Care. Antimicrobial use in Australian hospitals: 2016 annual report of the National Antimicrobial Utilisation Surveillance Program. Sydney: ACSQHC; 2018.

27. Bishop J, Kong DC, Schulz TR, Thursky KA, Buising KL. Meeting the challenge for effective antimicrobial stewardship programs in regional, rural and remote hospitals - what can we learn from the published literature? Rural Remote Health. 2018;18(2):4442.

28. Bishop JL, Schulz TR, Kong DCM, Buising KL. Qualitative study of the factors impacting antimicrobial stewardship programme delivery in regional and remote hospitals. The Journal of hospital infection. 2019;101(4):440-6.

29. Broom A, Broom J, Kirby E. Cultures of resistance? A Bourdieusian analysis of doctors' antibiotic prescribing. Social science \& medicine (1982). 2014;110:81-8.

30. Bishop JL, Schulz TR, Kong DCM, James R, Buising KL. Similarities and differences in antimicrobial prescribing between major city hospitals and regional and remote hospitals in Australia. Int J Antimicrob Agents. 2019;53(2):171-6.

31. eTG complete [Internet]. Melbourne: Therapeutic Guidelines Limited; 2015 Jul. https://tgldcdp.tg.org.au/etgAccess (date accessed: 5/09/2019).

32. Australian Commission on Safety and Quality in Health Care. Antimicrobial Stewardship in Australian Health Care 2018. Sydney: ACSQHC; 2018.

33. StataCorp. 2017. Stata Statistical Software: Release 15. College Station, TX: StataCorp LLC. 34. Zhao Y, Russell DJ, Guthridge S, Ramjan M, Jones MP, Humphreys JS, Carey TA, Wakerman J. Long-term trends in supply and sustainability of the health workforce in remote Aboriginal communities in the Northern Territory of Australia. BMC Health Serv Res. 2017;17(1):836.

35. Wright A, Briscoe K, Lovett R. A national profile of Aboriginal and Torres Strait Islander Health Workers, 2006-2016. Aust N Z J Public Health. 2019;43(1):24-6. 
467 36. Hawes LA, Turner L, Buising KL, Mazza D. Workflow-based data solutions are required to support 468 antimicrobial stewardship in general practice. BMJ Open Qual. 2019;8(3).

469 37. Thomas L, Bowen AC, Ly M, Connors C, Andrews R, Tong SYC. Burden of skin disease in two 470 remote primary healthcare centres in northern and central Australia. Intern Med J. 2019;49(3):396-9.

471 38. Australian Commission on Safety and Quality in Health Care (ACSQHC). AURA 2019:

472 supplementary data. Sydney: ACSQHC; 2019.

473 39. Hawes L, Turner L, Buising K, Mazza D. Use of electronic medical records to describe general 474 practitioner antibiotic prescribing patterns. Aust J Gen Pract. 2018;47(11).

475 40. Jongen C, McCalman J, Campbell S, Fagan R. Working well: strategies to strengthen the 476 workforce of the Indigenous primary healthcare sector. BMC Health Serv Res. 2019;19(1):910.

477 41. McCalman J, Campbell S, Jongen C, Langham E, Pearson K, Fagan R, Martin-Sardesai A, 478 Bainbridge R. Working well: a systematic scoping review of the Indigenous primary healthcare workforce 479 development literature. BMC Health Serv Res. 2019;19(1):767.

480 42. Wakerman J, Humphreys J, Russell D, Guthridge S, Bourke L, Dunbar T, Zhao Y, Ramjan M, 481 Murakami-Gold L, Jones MP. Remote health workforce turnover and retention: what are the policy and 482 practice priorities? Hum Resour Health. 2019;17(1):99.

483 43. National Safety and Quality Health Service Standards: User Guide for Aboriginal and Torres Strait 484 Islander Health, Australian Commission on Safety and Quality in Health Care. 2017.

485 44. Improving care for Aboriginal and Torres Strait Islander People. Resource 1. Setting safety and 486 quality goals for Aboriginal and Torres Strait Islander people in health service organisations. Australian 487 Commission on Safety and Quality in Health Care. 2016. 


\section{Table 1 (on next page)}

Descriptive characteristics of audit sample population.

${ }^{\text {A } W A: ~ K i m b e r l e y ~ r e g i o n ~ o f ~ W e s t e r n ~ A u s t r a l i a ; ~ N T: ~ T o p ~ E n d ~ o f ~ t h e ~ N o r t h e r n ~ T e r r i t o r y ; ~ Q L D: ~ f a r ~ n o r t h ~}$ Queensland

${ }^{\mathrm{B}} \mathrm{QQR}$ : Interquartile range 


\begin{tabular}{ccccc}
\hline & WA $^{\mathbf{A}}$ & $\mathbf{N T}^{\mathbf{A}}$ & QLD $^{\mathbf{A}}$ & Total \\
\hline Audit dates & $\begin{array}{c}02 / 03 / 2018- \\
02 / 06 / 2018\end{array}$ & $\begin{array}{c}28 / 08 / 2017- \\
03 / 09 / 2017\end{array}$ & $\begin{array}{c}10 / 05 / 2018- \\
15 / 06 / 2018\end{array}$ & $\begin{array}{c}28 / 08 / 2017- \\
15 / 06 / 2018\end{array}$ \\
\hline Unique patients & $186(35 \%)$ & $162(31 \%)$ & $181(34 \%)$ & 529 \\
\hline Antimicrobials used & $243(36 \%)$ & $196(29 \%)$ & $229(34 \%)$ & 668 \\
\hline $\begin{array}{c}\text { Antimicrobials used with } \\
\text { available microbiological } \\
\text { result }\end{array}$ & $36(15 \%)$ & $34(17 \%)$ & $82(36 \%)$ & $152(23 \%)$ \\
\hline $\begin{array}{c}\text { Age, years (median [IQR }]) \\
\text { Sex (M:F) }\end{array}$ & $22[5-44]$ & $24[8-46]$ & $23[8-44]$ & $23[7-45]$ \\
\hline $\begin{array}{c}\text { Aboriginal \& Torres Strait } \\
\text { Islander }\end{array}$ & $\begin{array}{c}169(91 \% ; 8 \\
\text { unknown) }\end{array}$ & $153(94 \%)$ & $\begin{array}{c}141(78 \% ; 10 \\
\text { unknown) }\end{array}$ & $463(88 \% ; 18$ \\
unknown)
\end{tabular}




\section{Table 2 (on next page)}

Frequency ( $(\%)$ ) of antimicrobial use by health professional, syndrome and antimicrobial.

${ }^{A}$ WA: Kimberley region of Western Australia; NT: Top End of the Northern Territory; QLD: far north Queensland

${ }^{\text {B } A \& T S I: ~ A b o r i g i n a l ~ a n d ~ T o r r e s ~ S t r a i t ~ I s l a n d e r ~}$

c SSTI: skin and soft tissue infection; GI: gastrointestinal infection; STI: sexually transmitted infection; LRTI: lower respiratory tract infection; RHD: rheumatic heart disease ${ }^{\mathrm{D}}$ Other: all syndromes/antimicrobials with frequency $<10 \%$ in every jurisdiction 


\begin{tabular}{|c|c|c|c|c|c|}
\hline & & $\mathbf{W A}^{\mathbf{A}}$ & $\mathbf{N T}^{\mathbf{A}}$ & $\mathbf{Q L D}^{\mathbf{A}}$ & Total \\
\hline \multirow{5}{*}{ 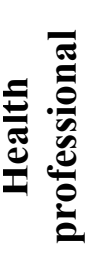 } & Doctors & $130(53 \%)$ & $51(26 \%)$ & $166(72 \%)$ & $347(52 \%)$ \\
\hline & Nurses & $100(41 \%)$ & $142(72 \%)$ & $63(28 \%)$ & $305(46 \%)$ \\
\hline & $\mathrm{A}^{2} \mathrm{TSI}^{\mathrm{B}}$ health practitioner & $1(<1 \%)$ & $3(2 \%)$ & $0(0 \%)$ & $4(1 \%)$ \\
\hline & Unknown & $12(5 \%)$ & $0(0 \%)$ & $0(0 \%)$ & $12(2 \%)$ \\
\hline & Total & $243(100 \%)$ & $196(100 \%)$ & $229(100 \%)$ & $668(100 \%)$ \\
\hline \multirow{7}{*}{ } & SSTI & $86(35 \%)$ & $57(29 \%)$ & $92(40 \%)$ & $235(35 \%)$ \\
\hline & GI & $28(12 \%)$ & $19(10 \%)$ & $19(8 \%)$ & $66(10 \%)$ \\
\hline & STI & $24(10 \%)$ & $6(3 \%)$ & $28(12 \%)$ & $58(9 \%)$ \\
\hline & LRTI & $20(8 \%)$ & $35(18 \%)$ & $8(4 \%)$ & $63(9 \%)$ \\
\hline & RHD & $6(2 \%)$ & $22(11 \%)$ & $14(6 \%)$ & $42(6 \%)$ \\
\hline & OtherD & $79(33 \%)$ & $57(29 \%)$ & $68(30 \%)$ & $204(31 \%)$ \\
\hline & Total & $243(100 \%)$ & $196(100 \%)$ & $229(100 \%)$ & $668(100 \%)$ \\
\hline \multirow{5}{*}{ 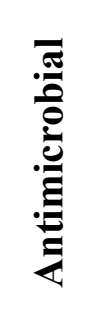 } & $\begin{array}{l}\text { Benzathine } \\
\text { benzylpenicillin }\end{array}$ & $37(15 \%)$ & $44(22 \%)$ & $31(14 \%)$ & $112(17 \%)$ \\
\hline & Amoxicillin & $27(11 \%)$ & $27(14 \%)$ & $24(10 \%)$ & $78(12 \%)$ \\
\hline & Co-trimoxazole & $16(7 \%)$ & $10(5 \%)$ & $42(18 \%)$ & $68(10 \%)$ \\
\hline & Other $^{\mathrm{D}}$ & $163(67 \%)$ & $115(59 \%)$ & $132(58 \%)$ & $410(61 \%)$ \\
\hline & Total & $243(100 \%)$ & $196(100 \%)$ & $229(100 \%)$ & $668(100 \%)$ \\
\hline
\end{tabular}




\section{Figure 1}

Map adapted from the Australian Bureau of Statistics, showing location and population of discrete Indigenous communities in northern Australia (i.e. above the Tropic of Capricorn (blue line)).

Source: Australian Bureau of Statistics, 2006, Housing and Infrastructure in Aboriginal and Torres Strait Islander Communities, cat. no. 4710.0, viewed 5 May 2020, https://www.abs.gov.au/AUSSTATS/abs@.nsf/Latestproducts/4710.0Appendix42006?opendoc ument\&tabname $=$ Notes $\&$ prodno $=4710.0 \&$ issue $=2006 \&$ num $=\& v i e w=$ 


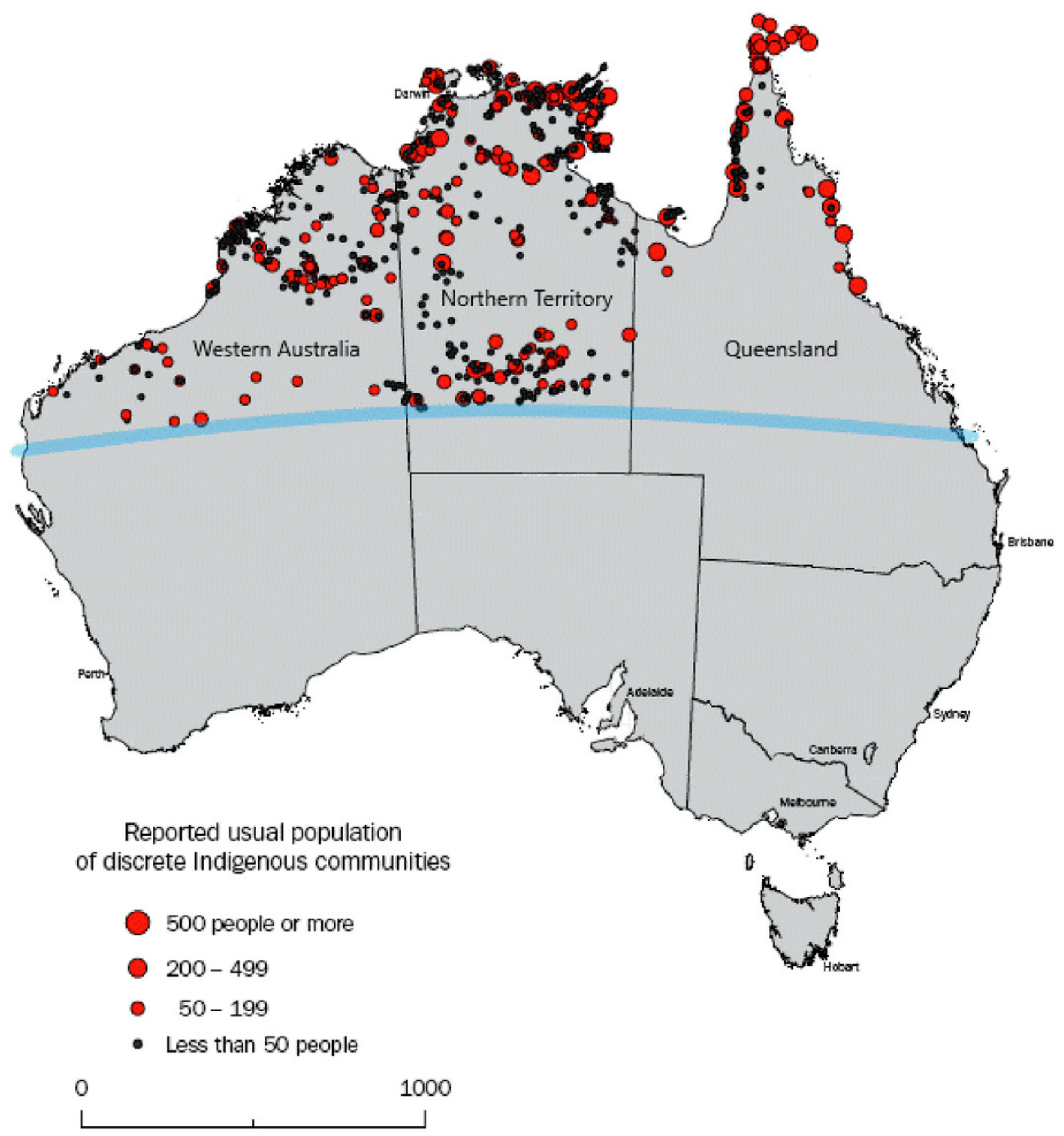

Kilometres 


\section{Figure 2}

Antimicrobial use by health professional and syndrome.

Frequency of antimicrobial use by health professional and syndrome in WA (Kimberley), the NT (Top End) and QLD (far north Queensland). Numbers in middle of columns are the number (not percentage) of antimicrobials used for that health professional/syndrome group. Total number of antimicrobials: WA - 230, NT - 193, QLD - 229. *Other: all syndromes with frequency $<5 \%$

WA

NT

antimicrobials by health professional (\%)

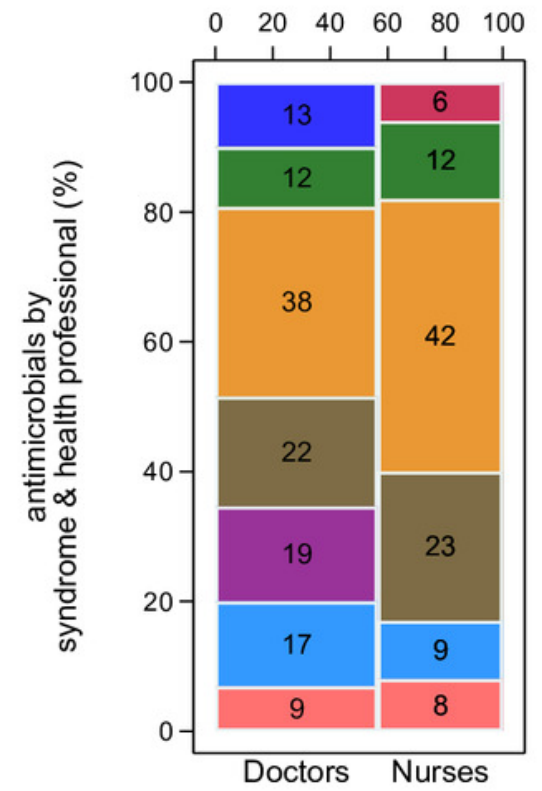

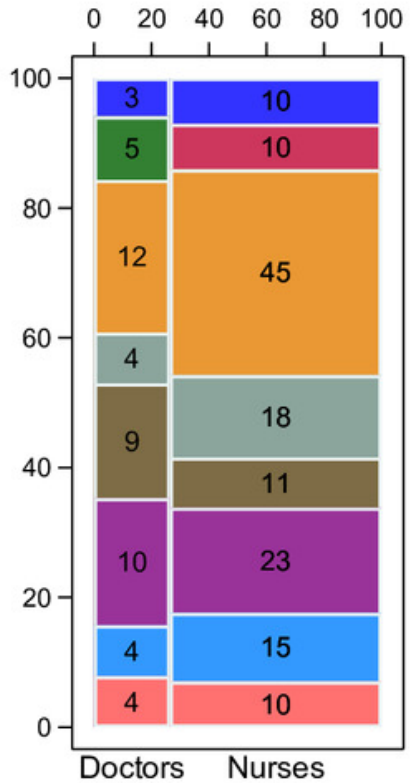

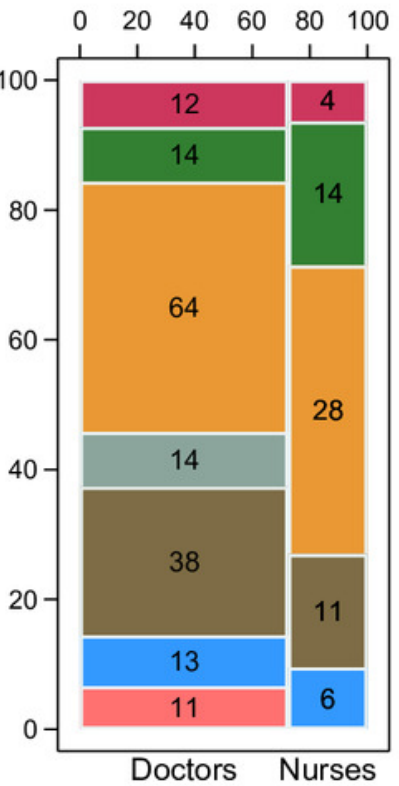

QLD

Doctors Nurses
Urinary tract infection

Skin \& soft tissue infection

Lower respiratory tract infection
Upper respiratory tract infection

Rheumatic heart disease

Gastrointestinal infection 


\section{Figure 3}

Antimicrobial use by health professional and antimicrobial for skin and soft tissue infections.

Frequency of antimicrobial use by health professional and antimicrobial for skin and soft tissue infections in WA (Kimberley), NT (Top End) and QLD (far north Queensland). Numbers in middle of columns are the number (not percentage) of antimicrobials used for that health professional/antimicrobial group. Total number of antimicrobials: WA - 80, NT - 57, QLD - 92. *Other: all antimicrobials with frequency $<5 \%$

WA

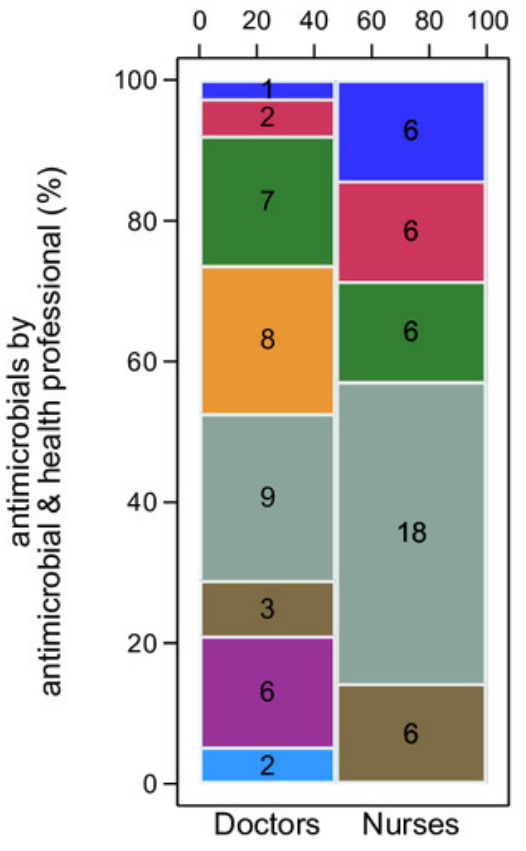

NT

antimicrobials by health professional (\%)

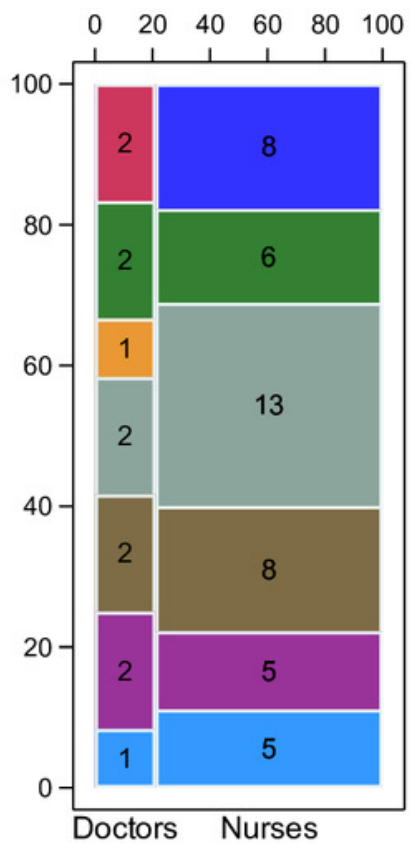

QLD

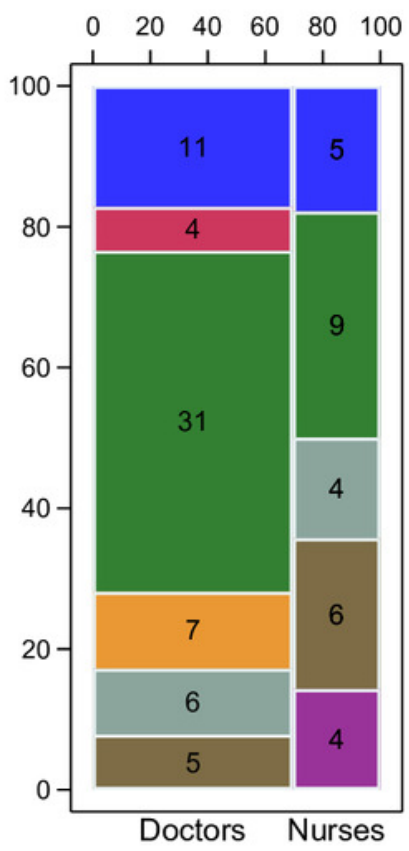

Other*

Benzathine benzylpenicillin
Flu(di)cloxacillin

Antiparasitic
Co-trimoxazole

Antifungal
Cephalosporin

Amoxicillin-clavulanate 


\section{Figure 4}

Appropriateness of antimicrobial use by doctors and nurses.

Appropriateness of antimicrobial use by doctors and nurses, by jurisdiction (WA (Kimberley), NT (Top End) and QLD (far north Queensland). Appropriate: Optimal or Adequate, Inappropriate: Suboptimal or Inadequate.

WA

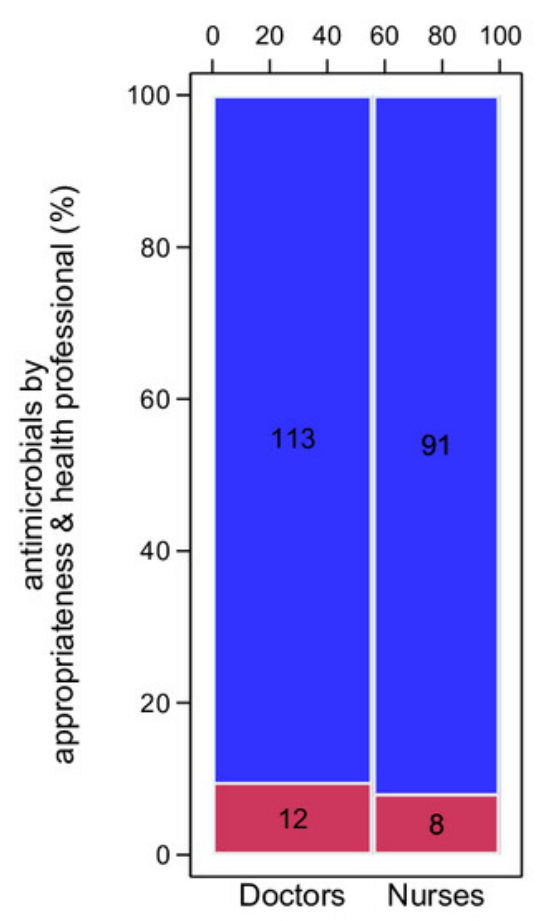

NT

antimicrobials by health professional (\%)

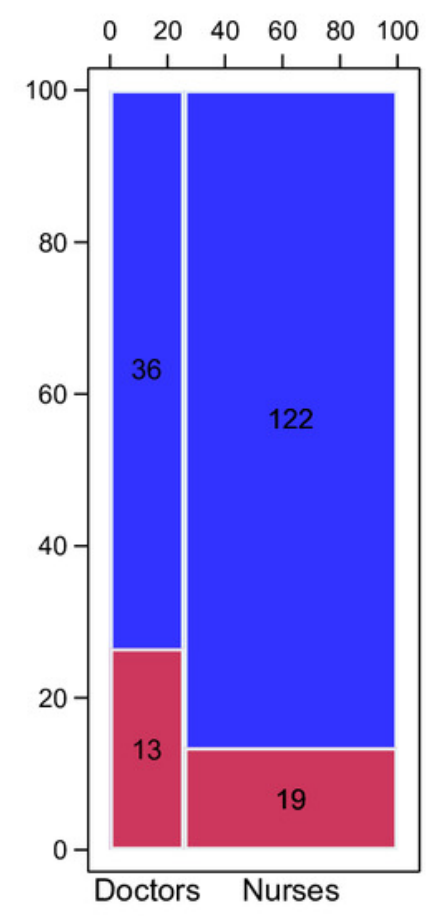

QLD

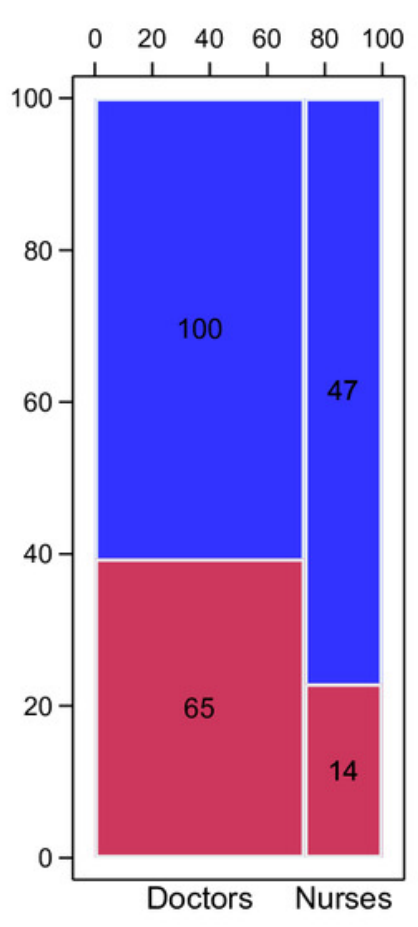

